\author{
Classification \\ Physics Abstracts \\ $52.50-52.75-41.80 \mathrm{G}$
}

\title{
UNE SOURCE D'IONS DE BASSE ENERGIE A FLUX ÉLEVÉ CHAUFFÉE EN HYPERFRÉQUENCES
}

\author{
Ph. BOULANGER, B. LE CLERC, J. L. LECLERC et G. MOURIER \\ Direction Technique Thomson CSF, Division Tubes Electroniques, \\ 2 , rue Latéocère, 78140 Vélizy Villacoublay, France
}

(Reçu le 3 mars 1977, révisé le 23 juin 1977, accepté le 28 juin 1977)

\begin{abstract}
Résumé. - Dans une décharge hyperfréquence, la pénétration du champ électrique permet d'obtenir une température électronique de 10 à $50 \mathrm{eV}$. Si la décharge est en contact avec une paroi ou une grille, un flux élevé d'ions est accéléré dans la gaine jusqu'à une énergie plusieurs fois supérieure à l'énergie thermique des électrons. Si le plasma peut se dilater librement le flux restera élevé mais son énergie n'atteindra qu'une fraction de cette valeur.

On décrit une étude expérimentale de ces phénomènes et l'application de la source ainsi constituée à l'érosion ionique d'une surface solide.
\end{abstract}

\begin{abstract}
In a microwave gas discharge, due to the penetration of the electric field the electron temperature can rise to 10 or $50 \mathrm{eV}$. If this discharge is in contact with a wall or a grid, a high ion flux is accelerated in the sheath, up to an energy several times the electron thermal energy. If the plasma is allowed to expand freely, the ion flux will remain high but its energy will attain only a fraction of the electron thermal energy.

Described is an experimental study of these phenomena, and the application of the source thus constituted to ionic etching of a solid surface.
\end{abstract}

1. Introduction. - Dans une décharge hyperfréquence, le champ électrique peut conserver dans le plasma une grande amplitude si la densité électronique n'excède pas la valeur critique. On peut alors obtenir une température électronique élevée et des effets de transport ambipolaire très importants. Ces décharges peuvent être excitées à basse pression $\left(10^{-4}\right.$ à $10^{-3}$ torr). Les ions subissent alors peu de collisions et peuvent se déplacer en véritables faisceaux accélérés à quelques dizaines d'électron-volts et neutralisés par les électrons (Fig. 1).

C'est sur cette base que J. L. Delcroix et J. Peyraud proposèrent [1] de construire des sources d'ions à basse énergie et grand courant, la loi de Child Langmuir ne s'appliquant plus à cause de la température élevée des électrons. Une étude théorique de ces auteurs a montré que les plasmas ainsi produits peuvent en outre être très fortement ionisés.

Nous nous sommes intéressés à cette décharge à cause des possibilités qu'elle offre pour le traitement

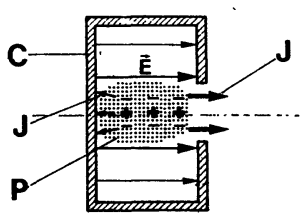

FIG. 1. - Principe physique d'une source C Cavité électromagnétique; P Plasma (positif); $E$ Champ électrique hyperfréquence; $J$ Courant ambipolaire d'ions accélérés suivant les électrons.

[Schematic diagram of a source : C, electromagnetic cavity; $\mathbf{P}$, plasma (positive); $E$, microwave electric field; $J$, ambipolar flow.]

des surfaces solides. Si l'on dispose de flux denses et étendus d'ions de quelques dizaines d'électrons-volts, on peut obtenir des effets de surface importants sans déranger la structure sous jacente. Toutefois, l'application présente des problèmes spécifiques : distance de la source à l'échantillon, possibilité de guider le flux par un champ magnétique, sans parler de la contamination etc. 
L'étude expérimentale d'un flux dense d'ions lents est difficile parce que ce flux s'arrête ou diverge par charge d'espace dès qu'on en retire les électrons. On doit donc multiplier les moyens de mesure pour s'assurer que l'on observe en effet tout le flux ionique. En outre, les électrons très énergétiques de ce type de décharge ionisent le gaz résiduel et créent une population d'ions lents qui donne d'importants courants impropres à l'érosion des surfaces. Enfin, la présence d'un champ magnétique variable nous a fait renoncer à utiliser un analyseur d'énergie.

Nous avons donc eu pour but principal de définir différents régimes de fonctionnement et de déterminer les conditions permettant de les obtenir. Les difficultés expérimentales ne nous ont pas permis d'étudier chaque régime en détail.

2. Études préliminaires. - 2. 1. ÉTUDES THÉORIQUES. - La littérature contient plusieurs travaux théoriques et des calculs numériques de grand intérêt pour cette étude.

Tonks et Langmuir [2] ont établi et discuté une équation intégro-différentielle qui régit dans certaines approximations le potentiel électrique d'un plasma unidimensionnel non collisionnel dont la loi de création est donnée. Harrison et Thompson [3] puis Caruso et Cavaliere [4] ont étudié des cas limites et notamment un régime ambipolaire ou de plasma où la charge d'espace nette est négligeable et où l'énergie cinétique communiquée aux ions sur un grand nombre de longueurs de Debye peut atteindre environ $0,5 k T_{\mathrm{e}}$; c'est le modèle supposé dans [1]. En outre, Self [5] a donné des solutions sans approximation sur la charge d'espace entre deux parois solides où le courant net est nul; elles correspondent à la formation d'une gaine d'une épaisseur de quelques longueurs de Debye et caractérisée par une chute de potentiel électrique de plusieurs $k T_{\mathrm{e}}$ qui détermine l'énergie des ions. Ce régime, que l'on peut appeler de gaine est le plus intéressant pour les applications qui demandent une énergie ionique élevée, à condition que l'on trouve le moyen d'extraire de la région d'ionisation un flux plus ou moins neutralisé suivant la distance sur laquelle on veut propager ce flux. La difficulté vient de ce que la gaine elle-même est presque vide d'électrons. En fait, on cherche des régimes intermédiaires entre les deux cas limites décrits d'une part par Caruso et Cavaliere, d'autre part par Self.

Nous avons trouvé des solutions approximatives avec deux grilles parfaites (discontinuité de champ électrique) puis avec une seule grille parfaite. Nous n'avons pas trouvé de solution numérique stable de l'équation de Tonks - Langmuir pour un plasma unidirectionnel non collisionnel créé dans une région limitée de l'espace et se propageant librement au-delà de la région de production, sauf avec au moins une grille.

2. 2. Études eXPÉRIMENTALES. - Une étude préliminaire a été effectuée afin de vérifier que l'on peut obtenir une température électronique beaucoup plus élevée que dans les colonnes précitées ainsi qu'un flux dense d'ions énergétiques. Elle a été effectuée avec de l'argon dans un guide d'ondes excité à $3000 \mathrm{MHz}$ (bande S) (Fig. 2), une puissance utile de 60 watts, un champ électrique d'environ $100 \mathrm{~V} / \mathrm{cm}$ s'étendant sur $2 \mathrm{~cm}$. On avait limité le plasma à un diamètre de $3,5 \mathrm{~cm}$ par une paroi diélectrique. Le circuit hyperfréquence est représenté sur la figure 3. On obtenait une décharge stable pour une puissance hyperfréquence incidente comprise entre 20 et 100 watts, et une pression voisine de $10^{-3}$ torr. Pour amorcer la décharge, il est toutefois nécessaire d'élever temporairement la pression.

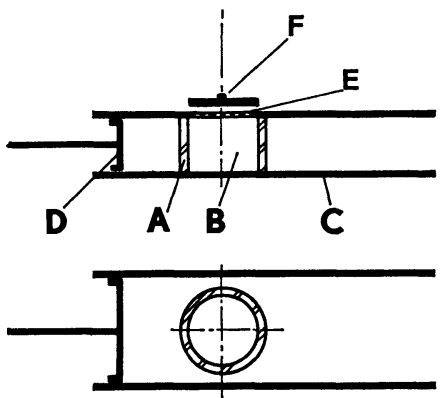

Fig. 2. - Guide ou cavité bande S. A Fenêtre étanche (diélectrique); B Zone de décharge; C Guide; D Piston; E Grille; F Collecteur.

[S band waveguide or cavity : A, vacuum tight dielectric window; $\mathrm{B}$, region of discharge ; C, waveguide ; D, tuning piston ; E, grid ; F, collector.]

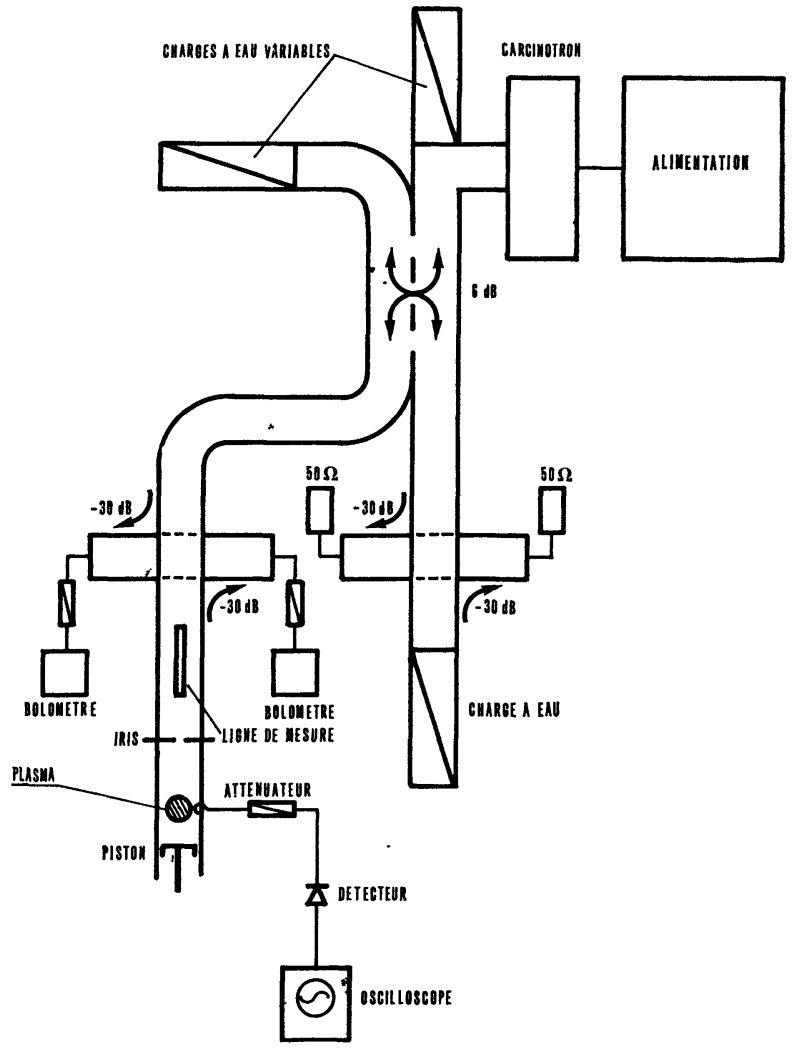

Fig. 3. - Schéma du circuit hyperfréquence.

[Diagram of microwave circuit.] 
La figure 4 montre la caractéristique d'un collecteur recevant le plasma par un petit trou pratiqué dans la paroi du guide. Ce n'est donc pas une caractéristique de sonde. Le trou étant petit, les conditions aux limites de Self (parois solides) sont à peu près remplies. On a représenté en pointillé une caractéristique théorique calculée d'après les résultats numériques de
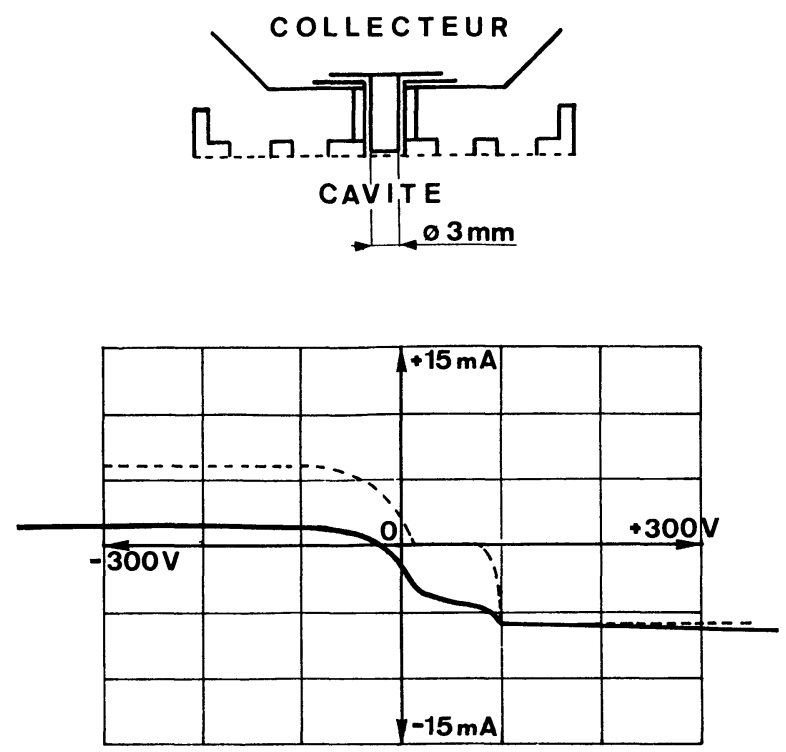

Fig. 4. - Caractéristique d'extraction à faible distance. Distance grille-collecteur $=0,5 \mathrm{~mm}$; Courbe expérimentale ; ----- Une courbe théorique $\left(T_{\mathrm{e}}=35 \mathrm{eV}\right)$. Origine des $V$ ajustée. $P=0,5 \times 10^{-4}$ torr.

[Extracted current characteristics at short distance $(0.5 \mathrm{~mm}$ between grid and collector) ; - Experimental curve ; --.-- A theoretical curve for $T_{\mathrm{e}}=35 \mathrm{eV}$. $V$-scale shifted. $P=0.5 \times 10^{-4}$ torr.]

cet auteur. La partie $V<0$, théoriquement exponentielle, donne une température électronique de $35 \mathrm{eV}$; le coude pour $V>0$ correspond à la répulsion des ions par le collecteur et devrait se produire d'après la théorie à $\mathrm{eV} \approx 5 k T_{\mathrm{e}}$ dans l'argon. On l'observe en fait à environ $100 \mathrm{eV}$, mais la charge d'espace ionique élevée pour $V>0$ peut expliquer la différence.

La densité de courant ionique au collecteur est de $20 \mathrm{~mA} / \mathrm{cm}^{2}$. Négligeant les effets de bord du trou, on peut en tirer d'après la théorie la valeur de la densité électronique au centre du plasma, soit $2 \times 10^{11} \mathrm{~cm}^{-3}$, alors que la densité critique (fréquence de plasma $=$ fréquence du champ) est de $1,1 \times 10^{11}$.

L'étude préliminaire a donc montré que le plasma créé par hyperfréquence permet d'atteindre à basse pression une température électronique $T_{\mathrm{e}}$ relativement très élevée, une densité supérieure à la densité critique, et un flux ionique caractérisé par une énergie de quelques $k T_{\mathrm{e}}$, une densité de courant élevée, un courant total exceptionnellement grand eu égard à l'énergie. Il s'agit de ce que nous avons désigné comme accélération de gaine.

3. Extraction du flux d'ions. - Si l'on veut utiliser le flux d'ions pour bombarder une surface solide, il est intéressant que son énergie soit limitée pour éviter la détérioration du solide en profondeur. Il est en outre souhaitable que le flux s'étende de la source à une distance de nombreuses longueurs de Debye; le flux, nécessairement neutre en courant total, doit en outre être localement neutre en charge d'espace. Nous avons montré qu'un système de deux grilles parfaites permet théoriquement d'y parvenir, mais la réalisation s'est montrée difficile. Il était nécessaire de construire une cavité plus élaborée (protection des isolants contre les métallisation, refroidissement, possibilités d'ajouter diverses grilles polarisées) et de plus grand diamètre, donc à fréquence plus basse.

3. 1. Cavité fonctionnant en Bande L (Fig. 5). - La cavité, de forme évasée possède une structure coaxiale avec un conducteur central plus court que le conducteur extérieur. Par raison de symétrie, cette structure est non radiative (même sans grille de fermeture, ce qui permet d'obtenir un faisceau de plasma étendu mais dont les ions sont peu énergétiques), et le champ électrique est uniforme à l'extrémité du conducteur central, dans l'axe de celui-ci. La décharge est excitée dans cette zone de champ uniforme à une fréquence d'environ $1270 \mathrm{MHz}$.

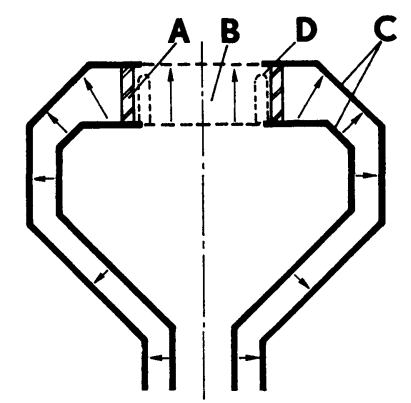

FIG. 5. - Cavité bande L. A Fenêtre étanche (diélectrique); B Zone de décharge; C Guide; D Cylindre conducteur ajouté ultérieurement.

[L band cavity : A, vacuum tight dielectric cylinder; $\mathrm{B}$, region of discharge; $\mathrm{C}$, waveguide; $\mathrm{D}$, location of conducting cylinder, a later addition.]

En ce qui concerne l'extension en longueur de la colonne de plasma créée dans la cavité, on cherche à s'affranchir de la limite correspondant à la longueur de Debye et à la distance maximale fixée par la loi de Child Langmuir pour les ions.

L'extraction à longue distance est facilitée par l'utilisation d'un champ magnétique parallèle à la direction du flux. La décharge dans la source est peu modifiée, le champ électrique étant parallèle à $B$, sauf si la fréquence cyclotronique des électrons est inférieure à environ le double de celle du champ électrique, auquel cas les phénomènes observés sont profondément modifiés par diverses résonances.

On a déjà souligné la difficulté de la mesure du courant ionique en présence de deux populations d'ions et d'électrons. Il est en outre difficile de régler la pression de gaz avec la précision nécessaire. 
3. 2. Mesure du fluX IONIQUe. - Il faut trouver les conditions dans lesquelles il existe des courants positifs élevés sur les électrodes et s'assurer qu'ils sont dus à un flux d'ions rapides et non à des ions thermiques accélérés dans les gaines de ces électrodes ou a des électrons secondaires émis par elles. En effet, la pression résiduelle de l'ordre de $10^{-4}$ torr dans le faisceau permet la création de ces ions thermiques.

Dans le but de réaliser les conditions de neutralité électrique permettant l'écoulement des ions, on a essayé de nombreux systèmes de grilles polarisées ou non entre la cavité et l'espace de glissement du faisceau. On a utilisé également un système en nid d'abeilles avec une maille de $5 \mathrm{~mm}$ pour créer une différence de pression entre la cavité et l'espace de glissement. Aucun pic d'ions rapides attendu n'ayant été observé, on a décidé de perfectionner les réglages (notamment de pression) et ne pas se limiter à des mesures électriques. Les moyens suivants ont été mis en ouvre :

- mesure de la pression dans une capsule recevant le flux d'ions par un orifice; guidé par le champ magnétique, le plasma pénètre librement dans la capsule où il se recombine sur les parois; il ressort sous forme de gaz mais son écoulement est freiné par l'orifice. Après détermination de la conductance de celui-ci à l'écoulement de gaz, la mesure de la pression donne le flux, égal au flux d'ions reçu;

- mesure simultanée de la température d'une cible calorimétrique mince à couple thermoélectrique et du courant qu'elle reçoit. On recherche les élévations de température accompagnées d'une pointe de courant positif. Ceci élimine les erreurs dues aux électrons secondaires.

En ce qui concerne le réglage de la pression, on a opéré en introduisant du gaz dans une réserve, en laissant la pression descendre en 20 minutes environ (Fig. 6 et 7), et en enregistrant simultanément la pression de l'enceinte et diverses grandeurs : courant de la cible et sa température, ou bien pression dans la capsule et courant, avec diverses grandeurs hyperfréquences.

La figure 6 illustre le fonctionnement de la capsule : 1) lorsqu'elle est polarisée à -10 volts par rapport à l'enceinte (Fig. 6A) sa pression intérieure $P_{1}$ varie comme la pression $P_{2}$ de l'enceinte en un point situé en aval;

2) pour une polarisation abaissée de 10 volts (Fig. 6B) on voit apparaître un pic correspondant à une augmentation de pression de $2,2 \times 10^{-4}$ torr alors que la pression de l'enceinte $P_{2}$ reste stable à quelques $\%$ près autour de $1,25 \times 10^{-4}$ torr. Si l'on attribue ce pic à une population d'ions ayant une énergie inférieure à $20 \mathrm{eV}$, le débit gazeux correspondant à cette population est, compte tenu de la conductance de l'orifice de la capsule calculée égale à $9 \mathrm{l} / \mathrm{s}$,

$$
9 \times 2,2 \times 10^{-4} \text { torr } 1 / \mathrm{s} \text { soit } 7 \times 10^{16} \mathrm{part} / \mathrm{s}
$$

soit encore un courant de $11 \mathrm{~mA}$.
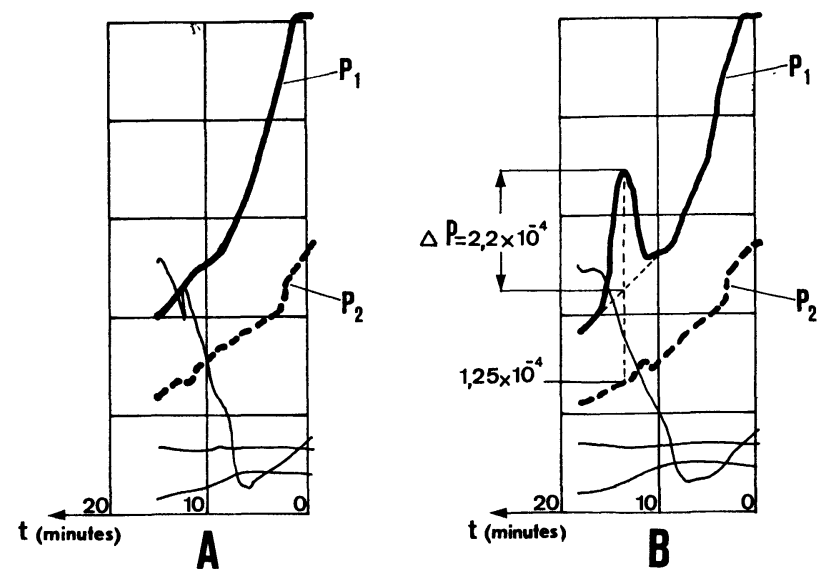

FIG. 6. - Enregistrements simultanés comprenant la pression dans la capsule. $A$ Polarisation de la capsule: -10 V. $B$ Polarisation de la capsule : $-20 \mathrm{~V} ; P_{1}$ Pression dans la capsule $\left(10^{-3}\right.$ torr pleine échelle); $P_{2}$ Prẹssion dans l'enceinte $\left(5 \times 10^{-4}\right.$ torr pleine échelle).

[Simultaneous recordings including pressure in the cell. $A$, cell potential : $-10 \mathrm{~V} ; B$, cell potential : $-20 \mathrm{~V} ; P_{1}$, pressure in cell $\left(10^{-3}\right.$ torr full scale); $P_{2}$, pressure in vessel $\left(5 \times 10^{-4}\right.$ torr full scale).]
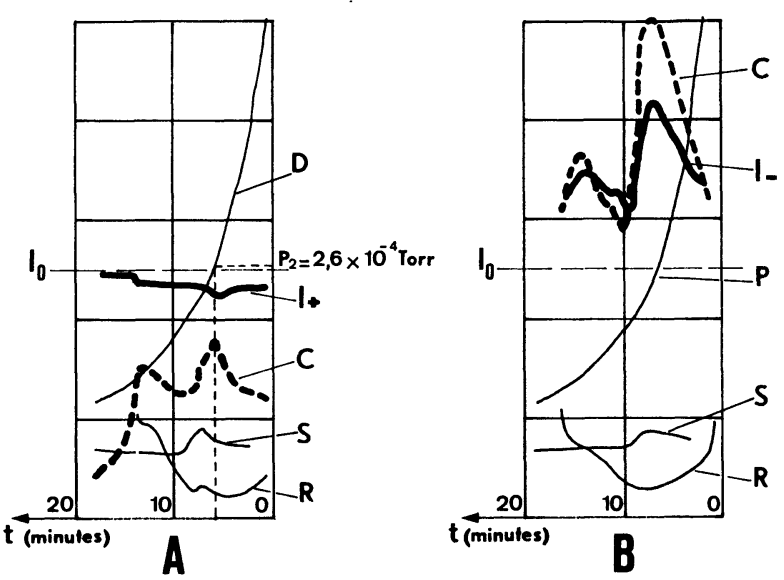

FIG. 7. - Enregistrement simultanés comprenant la température du calorimètre. $A$ Polarisation calorimètre: $-250 \mathrm{~V} ; B$ Polarisation calorimètre: $+250 \mathrm{~V} ; I_{0}$ Zéro de courant (échelle: $-12,5$ à $+12,5 \mathrm{~mA}) ; I_{+}$Courant ionique; $I_{-}$Courant électronique; $C$ Réponse du calorimètre $\left(460^{\circ} \mathrm{C}\right.$ pleine échelle); $R$ Puissance réfléchie (échelle: 0 à $300 \mathrm{~W}$ ); $S$ Réponse non calibrée d'une onde $\mathrm{HF}$ placée dans l'enceinte; $P$ Pression dans l'enceinte (échelle 0 à $5 \times 10^{-4}$ torr); Distance à la cavité : $18 \mathrm{~cm}$.

[Simultaneous recordings including temperature of the calorimeter. Calorimeter voltage : $A,-250 \mathrm{~V} ; B,+250 \mathrm{~V} ; I_{0}$, origin of currents (scale : $-12,5$ to $+12,5 \mathrm{~mA}$ ); $I_{+}$, ionic current ; $I_{-}$, electronic current ; $C$, calorimeter signal (460 ${ }^{\circ} \mathrm{C}$ full scale); $R$, reflected microwave power ( 0 to $300 \mathrm{~W}$ ) ; $S$, uncalibrated signal from a microwave probe close to calorimeter; $P$, pressure in vessel (0 to $5 \times 10^{-4}$ torr). Distance from cavity to calorimeter : $18 \mathrm{~cm}$.]

La section de l'orifice étant $0,4 \mathrm{~cm}^{2}$, la densité de courant est alors de $26 \mathrm{~mA} / \mathrm{cm}^{2}$.

La figure 7 illustre les résultats effectués avec la cible calorimétrique. On cherche les conditions de fonctionnement qui permettent de maximiser simulta- 
nément le courant ionique (négatif sur les courbes) et la température de la cible; on peut ainsi distinguer un courant ionique énergétique d'un courant d'électrons secondaires émis par la cible. Sur la figure 7B, la cible étant polarisée à $+250 \mathrm{~V}$, on ne voit pas apparaitre de telles conditions, alors qu'elles apparaissent sur la figure 7A (polarisation $-250 \mathrm{~V}$ ) dans deux régimes. Ayant ainsi déterminé la pression, on trace les caractéristiques de température et de courant collecté à la cible en fonction de sa polarisation électrique (Fig. 8).

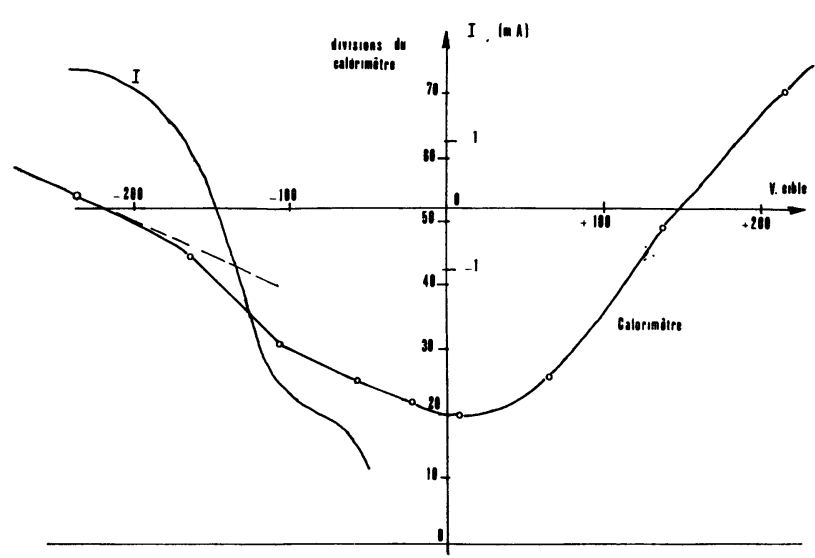

FIG. 8. - Caractéristiques du calorimètre.

[Calorimeter characteristics.]

Pour les fortes tensions positives ou négatives, le courant est purement électronique ou ionique et à peu près constant. Dans le cas de la figure 8 , le courant électronique de saturation n'a pas été enregistré mais il a été mesuré $(10 \mathrm{~mA})$. S'il existe des électrons secondaires, ils sont captés par la cible elle-même et ne faussent pas la mesure. Le courant étant constant, la lecture du couple de la cible, varie linéairement avec la tension. On utilise la partie électronique, où la mesure électrique est fiable, pour étalonner le calorimètre. Posant :

$$
\Delta N=k I \Delta V
$$

on obtient pour $k$ la valeur de $33 \mathrm{div} / \mathrm{W}$ (soit environ $150^{\circ} / \mathrm{W}$ ). Appliquant cette relation dans la région de saturation ionique, on obtient la valeur de $3 \mathrm{~mA}$ pour le courant. La valeur de la densité de courant ionique est alors de :

\section{$15 \mathrm{~mA} / \mathrm{cm}^{2}$}

Dans ces conditions, le courant lu est de $2 \mathrm{~mA}$, soit :

$$
10 \mathrm{~mA} / \mathrm{cm}^{2}
$$

La première valeur est par excès, la seconde par défaut, car la saturation ionique n'est pas tout à fait atteinte.

La cible calorimétrique ne permet pas de mesurer l'énergie des particules à cause de la gaine de potentiel qui se forme à son voisinage.

La mesure de courant ionique ainsi effectuée est beaucoup plus sûre que la mesure de flux par capsule manométrique car celle-ci n'est pas étalonnée et sa paroi interne peut désorber du gaz sous l'impact des ions.

Nous retenons comme sûre la valeur de $10 \mathrm{~mA} / \mathrm{cm}^{2}$. Le point important est qu'une telle densité de courant ne peut être transportée que par des ions fortement accélérés au voisinage immédiat de la région où ils sont créés, qui est nécessairement beaucoup plus étendue que la gaine formée au voisinage de la cible. En effet, il faut par exemple une densité de particules $6 \times 10^{10} \mathrm{~cm}^{-3}$ et une énergie de $20 \mathrm{eV}$, c'est-à-dire largement suprathermique. Le fait que le rapport des courants de saturation électronique et ionique est seulement de 5 en est une autre preuve. Il n'est pas exclu que la polarisation de la cible joue un rôle essentiel. Étant donné qu'elle est séparée de plus de $10 \mathrm{~cm}$ de la région de création des ions, ce ne peut être qu'en polarisant par rapport à la source, la colonne de plasma de façon à assurer sa neutralité électrique.

Il est ainsi démontré que l'on a obtenu une colonne à peu près neutralisée transportant un flux élevé d'ions d'environ $20 \mathrm{eV}$ sur plusieurs centaines de distances de Debye.

\section{3. CONDITIONS PERMETTANT D'OBTENIR UN FLUX} ÉLEVÉ À GRANDE DISTANCE. - Les mesures précédentes sont le résultat d'un grand nombre d'essais de différentes configurations de grilles polarisées au voisinage de la cavité hyperfréquence. Aux distances de 5 à $18 \mathrm{~cm}$, on n'a pu obtenir d'ions suprathermiques qu'en supprimant le nid d'abeilles (ce qui élève la pression dans l'enceinte à $5 \times 10^{-4}$ torr et favorise la formation d'ions thermiques) et d'utiliser une grille mince à pas très grand $(>2 \mathrm{~cm})$. Des grilles de même pas, épaisses dans la direction du flux, auraient l'avantage de réduire la pression résiduelle en avant, mais on a constaté qu'elles annulent le flux d'ions. On a effectué des mesures jusqu'à $18 \mathrm{~cm}$ de la source. Le courant ionique tombe alors à quelques $\mathrm{mA} / \mathrm{cm}^{2}$.

On constate que le flux ionique est absorbé par tout conducteur entouré d'une gaine de grande étendue, alors que le flux étendu obtenu, est justement caractérisé par l'absence de gaines et correspond au régime ambipolaire défini dans le paragraphe 2. 1. Cette interprétation est confirmée par des mesures sur une sonde mobile, montrant que le potentiel varie sur un très grand nombre de longueurs de Debye. La température électronique était généralement inférieure à $50 \mathrm{eV}$ et on n'observait pas d'érosion des cibles ce qui indique une énergie ionique probablement inférieure à $20 \mathrm{eV}$ [9].

Le tableau de la figure 9 permet de comparer les résultats obtenus d'une part avec le premier dispositif (paragraphe 1), où l'accélération se produisait dans la gaine, d'autre part avec la cavité en bande L, où elle était de nature ambipolaire.

4. Application de la source à l'érosion ionique. Avec le premier dispositif expérimental, fonctionnant 


\begin{tabular}{|c|c|c|}
\hline DÉSIGNATION & $\begin{array}{c}\text { CAVITÉ } \\
\text { BANDE S }\end{array}$ & $\begin{array}{c}\text { CAVITÉ } \\
\text { BANDE L }\end{array}$ \\
\hline Chauffage & $\mathrm{HF}$ & $\mathrm{HF}$ \\
\hline $\begin{array}{l}\text { Température électronique } \\
\text { Densité électronique }\end{array}$ & $\sim 20 \mathrm{eV}$ & $\sim 50 \mathrm{eV}$ \\
\hline dans la cavité & $\sim 10^{11} \mathrm{~cm}^{-3}$ & $\sim 2 \times 10^{10} \mathrm{~cm}^{-3}$ \\
\hline Pression & $\sim 2 \times 10^{-4}$ torr & $\sim 5 \times 10^{-4}$ torr \\
\hline Diamètre du faisceau & $2 \mathrm{~cm}$ & $7 \mathrm{~cm}$ \\
\hline $\begin{array}{l}\text { Densité du courant ionique } \\
\text { Énergie ionique }\end{array}$ & $\begin{array}{l}5 \mathrm{~mA} / \mathrm{cm}^{2} \\
\text { plusieurs dizaines } \\
\mathrm{d}^{2} \mathrm{eV}\end{array}$ & $\begin{array}{l}10 \mathrm{~mA} / \mathrm{cm}^{2} \\
\text { évaluée à } 10 \mathrm{eV} \\
\text { environ }\end{array}$ \\
\hline $\begin{array}{l}\text { Distance de diffusion } \\
\text { Homogénéité axiale } \\
\text { Homogénéité radiale }\end{array}$ & $\begin{array}{l}\text { inférieure à } 1 \mathrm{~cm} \\
\text { non mesurée }\end{array}$ & $\begin{array}{l}18 \mathrm{~cm} \text { au moins } \\
\text { bonne }\end{array}$ \\
\hline & & $\left\{\begin{array}{l}\text { bonne sur } \varnothing 2 \mathrm{~cm}, \\
\text { non mesurée plus } \\
\text { loin }\end{array}\right.$ \\
\hline Pas des grilles & $0,2 \mathrm{~mm}$ & $20 \mathrm{~mm}$ \\
\hline
\end{tabular}

FIG. 9. - Caratéristiques comparées des deux sources.

[A comparison between the two sources.]

en bande $S$, des mesures systématiques d'usinage ionique ont d'abord mis en évidence des régimes de décharge incontrôlés de faible énergie ionique. Nous avons effectué des érosions sur l'aluminium avec des vitesses d'usinage de l'ordre de $1 \mu / \mathrm{h}$. Avec un faisceau de $2 \mathrm{~cm}$ de diamètre, nous avons obtenu une érosion homogène radialement (Fig. 10), avec bords francs dans le cas de régime de décharge stable. Des accidents macroscopiques $(\simeq 1 \mu)$ apparaissent.

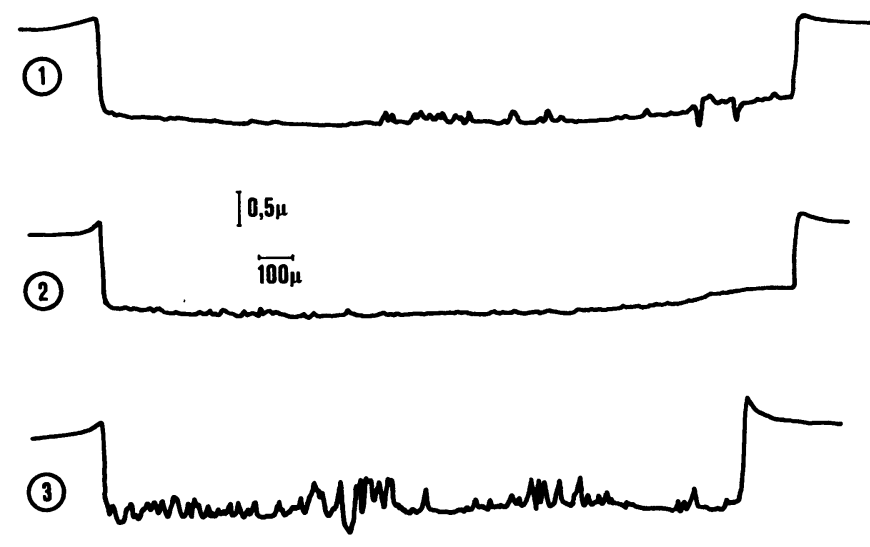

FIG. 10. - Enregistrement de la profondeur d'érosion. 1 à $6 \mathrm{~mm}$ du centre; 2 à $3 \mathrm{~mm}$ du centre; 3 centre de l'attaque.

[Recording of etching depth. Distance from axis : $1,6 \mathrm{~mm} ; 2,3 \mathrm{~mm}$; 3, on axis.]

Avec le système fonctionnant en bande $\mathrm{L}$, des essais infructueux ont conduit à une modification de la cavité où un cylindre métallique assurant une meilleure limitation du plasma a été mis en place (voir Fig. 5). Ce cylindre assure un bon contact avec le plasma dont le potentiel est alors mieux défini que par les grilles de la cavité. Dans ces conditions, on a effectué, après le réglage des potentiels permettant d'obtenir de l'érosion sur la cible, une trentaine d'essais suivis d'examens de la cible par diverses méthodes.
La température électronique était voisine de $23 \mathrm{eV}$.

La densité de courant ionique décroît très vite, en s'éloignant de la grille de sortie de la cavité. Les échantillons, placés à $1 \mathrm{~cm}$, recevaient une densité de courant de $0,3 \mathrm{~mA} / \mathrm{cm}^{2}$. La cible était la plupart $\mathrm{du}$ temps reliée à la masse; sa polarisation modifiait peu le courant reçu.

Des cibles d'aluminium et de $\mathrm{SiO}_{2}$ sur monocristal de Si ont été utilisées. La vitesse d'érosion était de 0,5 à $2 \mu / \mathrm{h}$.

On a utilisé des cibles de silicium orienté $<111>$ pour voir l'état du cristal après un bombardement ionique.

Il faut recourir à une méthode fine (pseudo lignes de Kikuchi) pour voir apparaître une couche amorphe à la surface de la zone usinée. Même si l'on ne prend en considération que la partie la plus en surface de la zone usinée en réduisant la tension d'accélération du diffractographe d'analyse à $3 \mathrm{kV}$, on ne distingue pratiquement pas de variation de structure cristalline entre la zone usinée et une zone masquée, alors que l'analyse au Dektak montre une érosion de $0,2 \mu$.

On a trouvé, par le spectre Auger, que la cible contient, après bombardement, 2,8\% d'aluminium en surface. La contamination en profondeur doit être nulle étant donné l'énergie des ions.

L'adjonction d'un champ magnétique élevé ( $>1000$ gauss) augmente le courant obtenu sur la cible et guide le faisceau; la figure 11 montre une

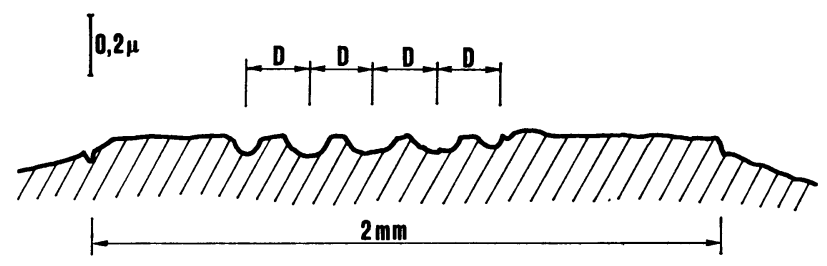

Fig. 11. - Enregistrement du profil de la surface. On retrouve le pas de la grille $(D=0,2 \mathrm{~mm})$ sur l'échantillon, situé à $5 \mathrm{~mm}$ de celle-ci.

[Recording of surface profile, reproducing the grid mesh $(0.2 \mathrm{~mm})$. Distance from grid to surface : $5 \mathrm{~mm}$.]

image de la grille de sortie de la cavité (pas 0,2 mm). Un champ magnétique plus bas permet des résonances cyclotroniques qui changent complètement la nature des décharges; on observe alors fréquemment sur la cible des dépôts des métaux constituant la cavité (Fig. 12).

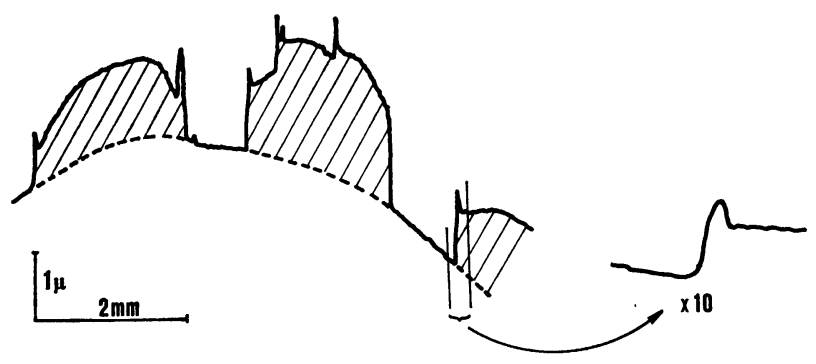

FIG. 12. - Enregistrement du profil des dépôts.

[Recording of the profile of a deposit.] 
La figure 13, obtenue au microscope à balayage (X 5000 ) donne le bord d'une zone masquée après 55 minutes d'exposition à $6,5 \mathrm{~mm}$ de la grille.

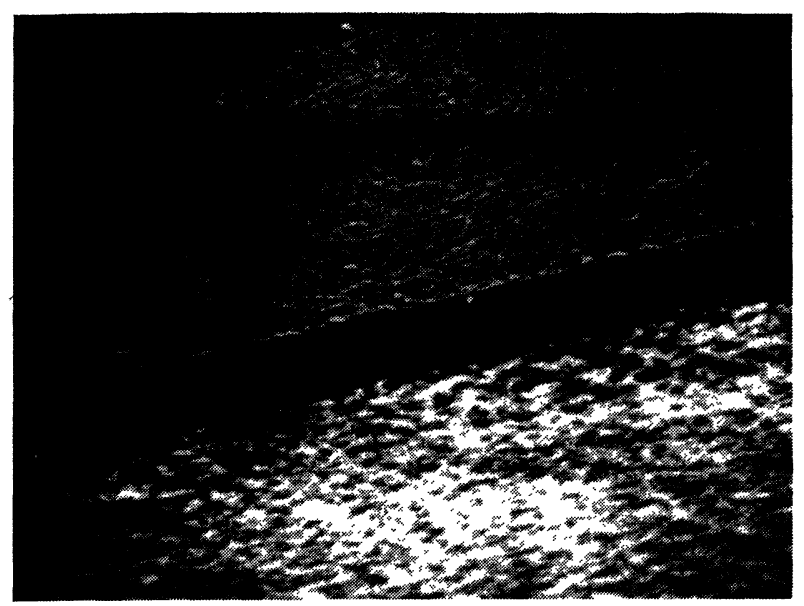

Fig. 13. - Examen de la surface au microscope à balayage. $\times \sim 5000$

[Sweeping electron microscope view of the edge of etching $(\times 5000) \cdot]$

5. Conclusions. - Nous avons montré dans une première expérience que les décharges chauffées par champ hyperfréquence permettent d'obtenir des températures électroniques élevées et des flux d'ions non limités par la loi de charge d'espace de Child Langmuir.

Ces sources ne semblent pas limitées en diamètre, si l'on met au point un circuit hyperfréquence de grandes dimensions en ondes progressives.

On pourrait naturellement accélérer ensuite ce flux d'ions de manière conventionnelle. Nous avons cherché à produire des flux neutralisés à basse énergie suivant diverses méthodes caractérisées essentiellement par les grilles qui limitent la cavité hyperfréquence et leur polarisation ou éventuellement par l'absence de grilles.

Dans une seconde série d'expériences, nous avons trouvé des conditions qui permettent d'obtenir un flux d'ions $\mathrm{A}^{+}$d'environ $10 \mathrm{~mA} / \mathrm{cm}^{2}$ sur une grande distance $(18 \mathrm{~cm})$ avec une énergie ionique de 15 à $20 \mathrm{eV}$, et nous pensons que ce flux est de type ambipolaire. Pour obtenir dans ces conditions des effets d'érosion, il faudrait pouvoir augmenter la température électronique, ce que nous n'avons pas réussi à faire par des réglages hyperfréquences.

Enfin, nous avons déterminé les conditions où l'énergie ionique est suffisante pour obtenir des effets d'érosion importants sur une cible solide. Il n'a pas été possible jusqu'à présent d'obtenir ces effets sans utiliser entre la cavité hyperfréquence et la cible, proche de 0,5 à $1 \mathrm{~cm}$, un système de deux grilles polarisées. L'énergie plus élevée des ions tient donc à la formation de gaines et ce régime ressemble aux cas unidimensionnels calculés par Self.

En ce qui concerne les effets d'érosion, la vitesse est comparable avec celle des machines couramment utilisées. Toutefois, l'énergie faible des ions évite divers effets de détérioration des cibles traitées. Le mode d'érosion ainsi offert est donc en compétition avec d'autres décharges haute fréquence. Les avantages respectifs, variables suivant les cas spécifiques, peuvent tenir à divers facteurs :

- pression de neutres basse, surtout si le faisceau peut être guidé à distance;

- impuretés venant des électrodes (éliminer les grilles); lité);

- possibilités de transporter le flux d'ions (neutra-

- géométrie de la décharge (absence d'électrodes dans l'enceinte, possibilités de systèmes étendus ou non, etc.).

Nous cherchons d'abord à nous affranchir des grilles et à transporter le faisceau à plus grande distance, ce qui est possible d'après la théorie ambipolaire.

Remerciements. - Nous remercions tout particulièrement la D.R.M.E. pour son soutien efficace et son intérêt pour ce travail, ainsi que MM. J. L. Delcroix, J. Peyraud et Y. Archambault pour de nombreuses discussions à tous les stades de certravail.

\section{Bibliographie}

[1] Delcroix J. L., Peyraud J., Rapport D.R.M.E. 22.06.73.

[2] Tonks L. et Langmuir I., Phys. Rev. 34 (1929) 876.

[3] Harrison E. R. et Thompson W. B., Proc. Phys. Soc. 74 (1959) London, 145.

[4] Caruso A. et Cavaliere A., Nuovo Cimento 26 (1962) 1389.

[5] Self S. A., Phys. Fluids, 6 (1963) 1762, 36 (1965) 456.
[6] Gurevitch A. V., Pariskaya L. V., Pitaevski L. P., Sov. Phys. JETP 22 (1966) 449, Sov. Phys. JETP 36 (1973) 274.

[7] Crow J. E., Auer P. L., Allen J. E., J. Plasma Phys. 14 (1975) 65.

[8] Bezzerides B. et Du Bors D. F.. Phis. Rev. Lett. 34 (1975) 1381.

[9] WeHNER G. K., dans Le bombardement ionique (Éditions du CNRS), Paris 1962. 